(C)2007 IEEE. Personal use of this material is permitted. However, permission to reprint/republish this material for advertising or promotional purposes or for creating new collective works for resale or redistribution to servers or lists, or to reuse any copyrighted component of this work in other works must be obtained from the IEEE 


\title{
Reconfigurable Software Architecture for Voice Access to Data Services
}

\author{
Alex Talevski ${ }^{1}$ and Elizabeth Chang $^{2}$ \\ ${ }^{1,2}$ DEBI Institute, Curtin University, GPO Box U1987, Perth, WA, 6845, Australia \\ e-mail : (alex.talevski, elizabeth.chang)@cbs.curtin.edu.au
}

\begin{abstract}
The rapid growth of computing, the Internet and telecommunications systems have resulted in a broad range of ways to communicate and access information. Telecommunication and data convergence promises a wide range of solutions that will increase productivity, reduce costs, and provide new opportunities and revenues for enterprises. Unfortunately, the access of converged telecommunication and data services has been isolated to specialised inflexible environments.

Due to the versatile nature of multimedia convergence, an adaptable approach is required. True voice and data convergence must be accessible anywhere and anyhow. This paper presents a reconfigurable software architecture driven solution that dynamically composes, integrates and tailors voice plugins. The solution offers enhanced service effectiveness, flexibility and convenience for professionals on the move.
\end{abstract}

Index Terms-Voice and Data Convergence, Reconfigurable Software, Computer Telephony Integration (CTI), Interactive Voice Response (IVR), Voice over Internet Protocol (VoIP)

\section{INTRODUCTION}

We live in a dynamic, fast-paced world. The advent of the web and powerful mobile devices has provided mechanisms for communication and information sharing anywhere $[1,2]$. Now, more than ever, information exchange, negotiations and decisions are performed away from the office. Enterprises have to consider the growing population of mobile stakeholders that would benefit from the next generation of converged telecommunication and data services. Flexible, rich access to enterprise services for its employees, partners and customers is essential.

However, to date, such convergence has been largely isolated to static environments where fixed Personal Computers (PC), telecommunication devices and Internet connections are used. Furthermore, data available on enterprise applications are not available on the telecommunications networks and devices and vice-versa.

The remainder of this section outlines the proposed solution background. It discusses Computer Telephony Integration (CTI), the Voice over Internet Protocol (VoIP) and Interactive Voice Response Telecom (IVR). Section 2 details the Reconfigurable Voice Access to Data (RVAD) solution. Section 3 illustrates the adopted Meta model. Section 4 outlines the implementation and third party tools. Section 5 concludes the paper.

\section{A. Computer Telephony Integration (CTI)}

Telephone and computer systems are two technologies that impact many aspects of our daily lives. These technologies drive the world's economy and are central to the operation of virtually every enterprise. Numerous organizations exist only with the support of these technologies.

Computer Telephony Integration (CTI) incorporates computers and telephony systems [1]. Computer features such as data handling, media processing and graphical user interface are combined with telephone features such as call handling and routing. Currently CTI is predominantly used to drive software-based Private Automatic Branch eXchanges (PABX).

\section{B. Voice over Internet Protocol (VoIP)}

Voice over Internet Protocol (VoIP) (also termed IP Telephony) is a CTI solution that is commonly used as an alternative telecommunication medium. Generally, VoIP refers to the transport of voice traffic over a data network where hardware and software act as an Internet transmission medium for telephone calls.

VoIP is particularly useful when there is limited or financially prohibitive access to alternative telephony networks. Telephone calls can be transmitted with little or no loss in functionality, reliability, or voice quality.

Using VoIP and software driven PABXs, CTI has provided access to telephony services through computer applications that provide feature rich and customized environment that is cheaper to run while being more productive.

\section{Interactive Voice Response Telecom (IVR)}

Interactive Voice Response Telecom (IVR) systems provide computer controlled telephone answering and routing functions, as well as facilities for the collection and provision of information. Interactive menus allow callers to input data using the telephone keypad and/or simple voice prompts. IVR devices route calls to the appropriate place or data, based on user defined steps, commands and responses to prompts. These systems may use a mixture of human and computer interaction which is provided live, pre recorded by an attendee or digitally synthesized sound to convey data to the caller.

Unfortunately, industry has failed to make the most of CTI, VoIP and IVR technologies. Current use of these technologies has been limited to telephony applications like telephone switchboards and conferencing. Existing systems rarely provide an integrated approach where more than communication services are provided. A flexible approach that integrates the features of these technologies is required. 


\section{ReConfigurable Voice ACCess to Data (RVAD)}

Traditionally, enterprise applications allow users to access corporate data in a static location using a personal computer. However, today's business professionals have a busy schedule and they are frequently on the move. Telecommunications and data convergence is required as a consequence of the increased flexibility that businesses demand [3].

The RVAD system eliminates traditional barriers by offering a new and novel solution that allows flexible access to a range of enterprise and internet services on the move. It provides the glue between enterprise business applications and multi-modal forms of business communication. The system is employed to access a variety of telecommunications and data services with or without the use of a personal computer or an internet connection.

An Interactive Voice Response (IVR) system is programmed using a reconfigurable plug and play software architecture [4] to dynamically compose, integrate and tailor voice access to data services. Desired content is then delivered direct to devices in an audible format within the context of a single converged telephone conversation. System interaction is performed using voice commands or touch-tones.

\section{A. RVAD Architecture}

The ability of software to adapt or be adapted to changes in environment is referred to as the software's flexibility [5]. Flexible solutions are needed due to the requirement for software to be deployed in diverse roles and environments (its diversity) and to be flexible to unclear, lacking and/or evolving information regarding these roles and environment (uncertainty) [5]. Software systems are versatile if they exhibit generic and function rich properties.

Components are the smallest possible reusable software modules that are of independent production, acquisition, and deployment [6]. Components partition a system into a number of well-defined boundaries in order to reduce software complexity and facilitate software evolution [7]. A component's services are the attributes and operations that a component provides. Such services are provided through the component interface using a loosely coupled messagebased communication model.

A component implemented by combining the functionality provided by other components is referred to as a composite component and the process of developing component composites as component composition. Component composition can be seen as a way to master software complexity [7]. Composite components are incrementally built out of lower-level components using an iterative process. Using this approach, increasingly complex applications are created by progressively composing components.
Research has shown that component-based software engineering leads to software that exhibits higher quality, shorter time-to-market and therefore, lower development cost $[8,9,10]$. However, it is difficult to modify a component-based system that has been statically composed during development. Re-configuration, addition, removal or replacement of components may require significant changes to the application source-code. Such changes have proven to be error-prone, time consuming and expensive.

In order to develop a solution to the issues outlined above and construct a solution from disparate components, a reconfigurable plug and play component-based architecture has been employed [4].

This RVAD architecture promotes simplified software construction, customisation, integration and evolution. It constitutes the skeletal support that is used as the basis for constructing composite component architectures from interconnected components. The architecture makes it possible to easily integrate existing enterprise and internet applications and newly implemented components as voice plugins.

Customised voice plugins are constructed by mixing and matching voice components, interaction engines and data source components. It is possible to tailor specific compositions and restrict the usage of components as required.

\section{B. RVAD Voice Plugin Host}

An RVAD voice plugin host behaves as an IVR entrypoint. As illustrated below (Figure 1), voice plugin discovery, query, identification and invocation are used to situate, define, describe and utilize available services. Once a voice plugin has been identified it is assigned to a telephone number. A user enters this number using Dual Tone Multi Frequency (DTMF) tones to invoke a voice plugin's services. Upon activation, and during execution, each voice plugin governs user interaction and the provision of its services.

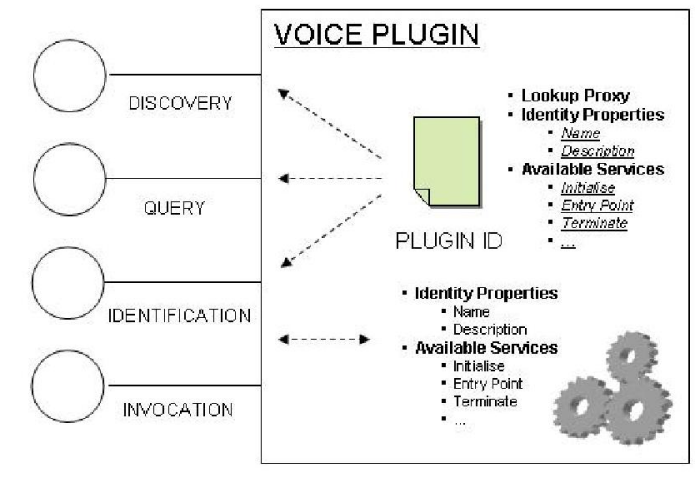

Fig. 1: Voice Plugin 


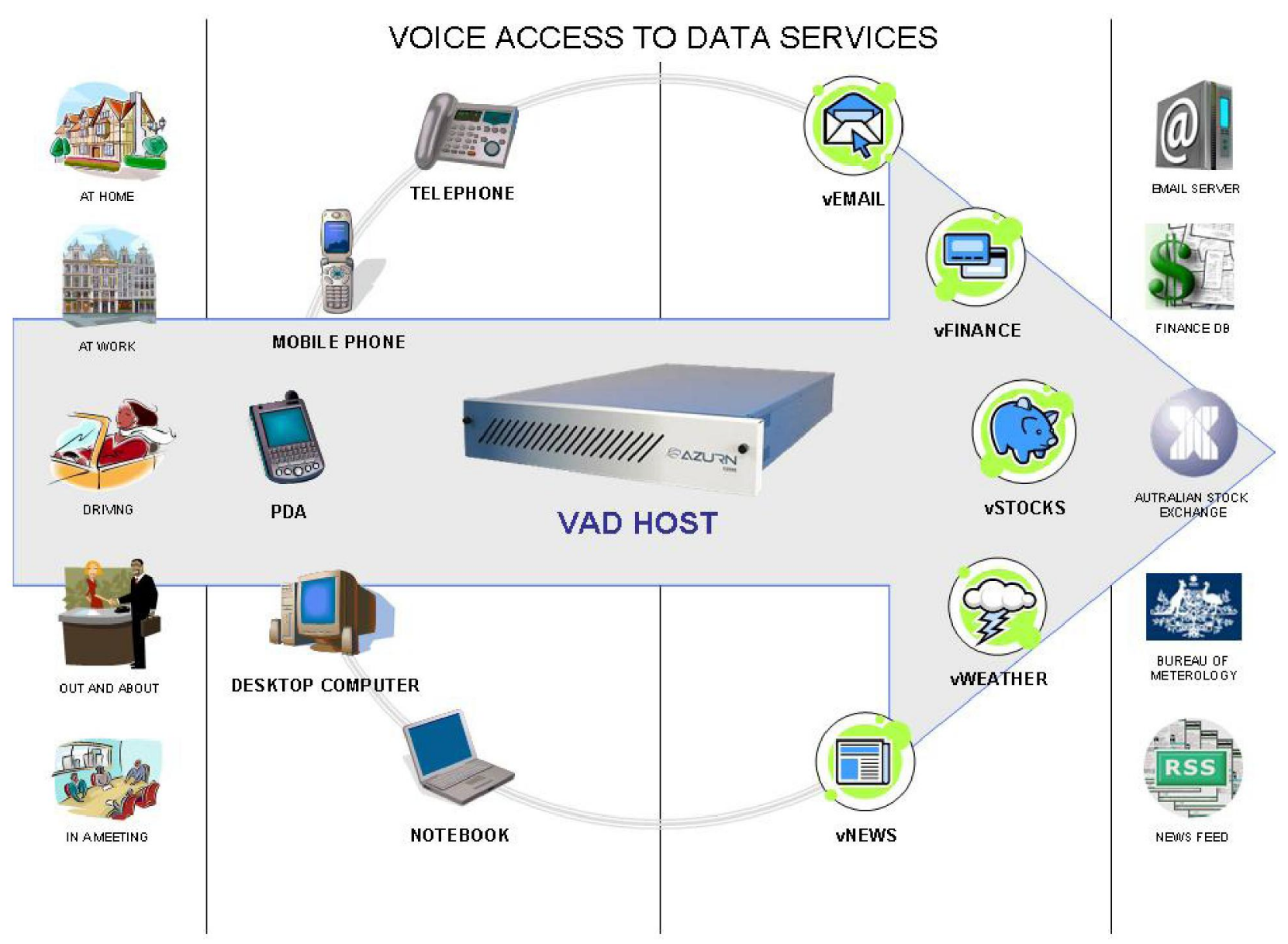

Fig. 2: Reconfigurable Voice Access to Data (RVAD)

The plugin architecture defines the following component interface;

- Plugin Discovery - Plugin discovery is used to find, identify, describe and use available voice plugins. In order to locate plugins, the RVAD server broadcasts a request for a voice plugin lookup service. Each voice plugin responds to this request with a lookup proxy.

- Plugin Query - The RVAD Plugin Host is able to query the voice plugin lookup service for available services.

- Plugin Identification - The voice plugin lookup service is used to define voice plugin characteristics.

- Plugin Invocation - When a voice plugin is selected via the IVR the RVAD Plugin Host dynamically binds to the voice plugin and invokes its entrypoint. The voice plugin then takes over interaction control and performs its identified services.

Figure 2 illustrates a high level composition of the RVAD system with sample voice plugin services (vEmail, vFinance, vStocks, vWeather, and vNews).

\section{Meta Model}

This solution makes use of explicit Meta component interface specifications and compositions [8]. Components, their interfaces and their interconnections are defined as explicit Meta data. This Meta model serves to define an application's composition. Such a composition is formed from interconnected components that are constructed in a layered and hierarchical manner where components at the lower layers satisfy the requirements of those above them.

The Unified Modelling Language (UML) is used to illustrate the architecture used to construct the RVAD solution.

\section{A. Components}

The component Meta data model illustrated in (Figure 3) is an explicit specification of a single component and its interface. Developers use this Meta data in order to evaluate the services that a component consumes and provides. Component interfaces define the possible component interactions. Available attributes and operations are exposed via the component interface. 


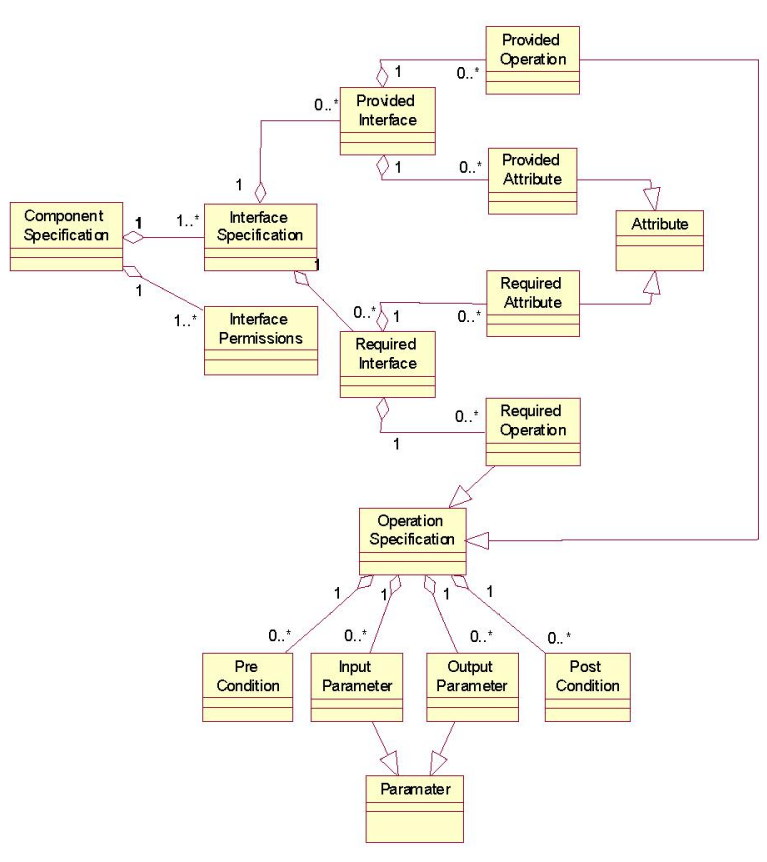

Fig. 3: Conceptual Meta Model of a Component

\section{B. Component Interaction and Collaboration}

Component collaborate with others through their interconnections. Such interconnections denote the specific component associations through a consumer / provider relationship. Component coordination and interaction is performed using message exchanges.

Connectors provide a level of indirection that reduces dependencies among components. Compositions are constructed using operation and attribute connectors to form a composite architecture. Attribute and operation connectors are used to directly connect components required and provided services. Once connected, the required interfaces of one component may be satisfied by the provided interfaces of another. Explicit invocations can be performed once two interfaces are connected [5]. These invocations are modelled on the requestor's side, by out / in (solicit / response) operations that correspond to in / out (request / reply) operations on the provider's side [11]. Multiple connectors, connector types and intermediate components are used in order to compose a fully composed RVAD solution.

\section{Attribute and Operation Connectors}

Figure 4 illustrates a Meta model of a component attribute connector and component operation connectors [12]. Attribute and operation connectors are used to connect required and provided RVAD attributes and operations.

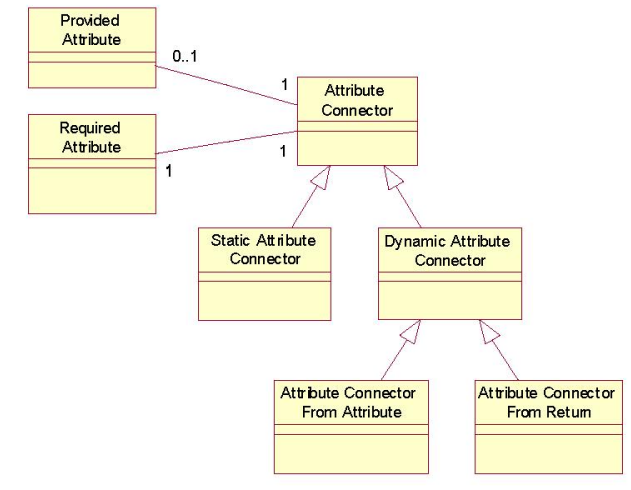

Fig. 4a: Meta Model - Attribute Connector

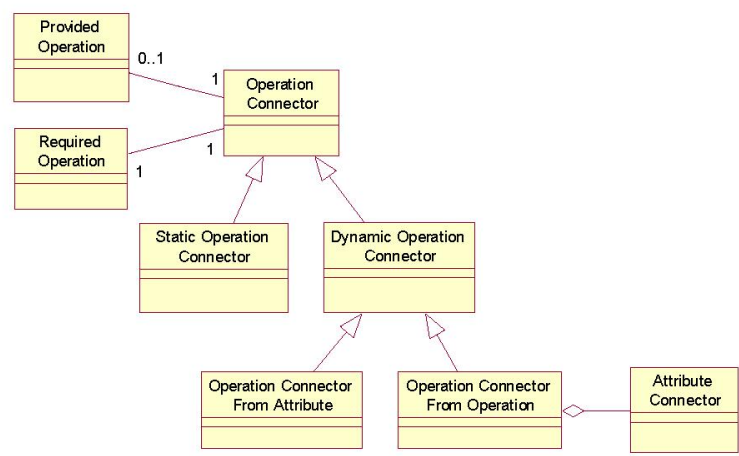

Fig. 4b: Meta Model - Operation Connector

\section{IMPLEMENTATION AND THIRD PARTY TOOLS}

The software platform is an N-Tier distributed computing architecture that employs Enterprise Java Beans (EJB), Java 2 Enterprise Edition (J2EE), and XML technologies. The RVAD solution employs an interaction Finite State Machine (FSM) to govern voice plugin services. The System can be broken into the following subsystems;

- Login and session management is provided by an authentication component. This component provides authentication and session validation functions.

- The voice interface component is responsible for all user interactions.

- Audio encoding/decoding is performed by a voice data component.

- Voice XML (VXML) is encoded/decoded using a voice document component. This component is responsible for the creation, parsing and programmatic manipulation of voice documents.

- The database component queries and persists all required input and output. 


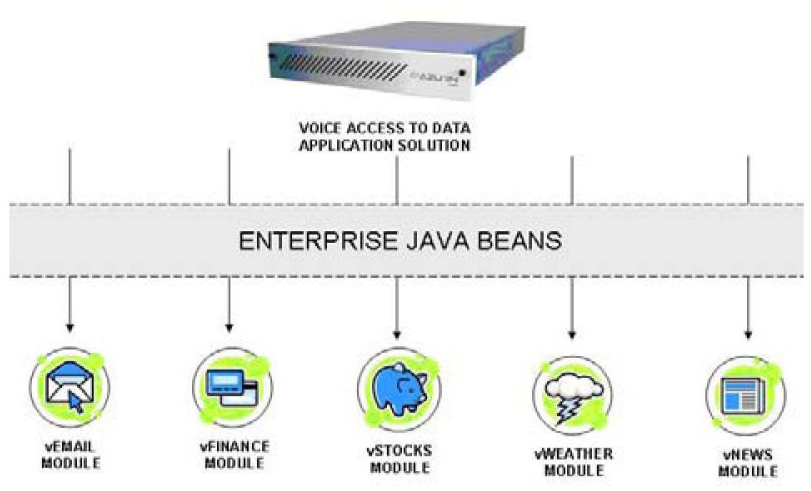

Fig. 5: Application Solution

The RVAD application (Figure 5) is constructed from a number of lower level voice plugins that are fully composed business modules. The diagram below illustrates the high level RVAD application solution with a set of sample business modules.

Business modules (Figure 6) represent voice plugins that typically perform a single voice access to data service. Such business modules utilise groups of component compositions. The high-level diagram below illustrates the vEmail business module. This business module is constructed from multiple lower-level generic components which are reused in other business module compositions.

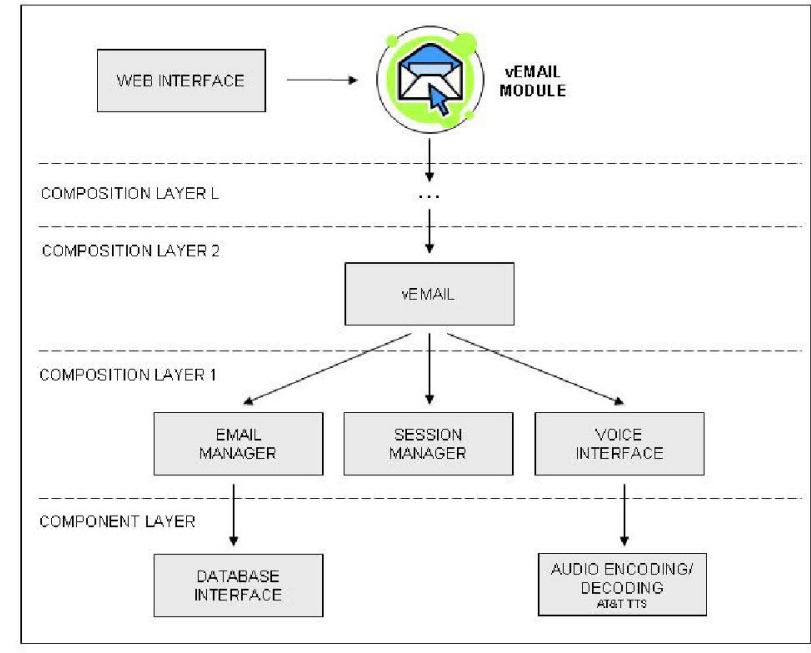

Fig. 6: Business Module

Customised voice plugins are constructed by mixing and matching hierarchically interconnected components

\section{E. Existing Voice Plugins}

- vEMail Service - Emails can be accessed at any time and followed up instantly. The vEMail voice plugin hosts a Post Office Protocol (POP) email service where high priority emails can be forwarded for voice access. The vEMail voice plugin reads out each email using a clear voice. The user may interact with the vEMail voice plugin by telephone key tones. RVAD allows the automatic browsing of emails without user intervention. It is possible to reply to emails with predefined email templates and forward messages to predefined contacts immediately. Users may manage their email messages by saving, moving and deleting selected items. It is also possible to customize the way that the system performs and to manage emails contacts and template messages via an easy to use web interface.

- vStocks Service - Live Australian Stock Exchange (ASX) values can be heard at the user's convenience. The vStocks service reads out detailed information on each user's individually predefined stocks. Stock list navigation is performed using telephone key tones. RVAD allows the browsing of stock data without user intervention. The vStocks service is able to announce each stock's trade date, time, change, previous close, day high, day low, and volume. Users may customize the stock properties they wish to hear to suit their individual preferences.

- vWeather Service - Live Bureau of Meteorology $(\mathrm{BOM})$ weather forecasts can be accessed at any time. The vWeather service reads out detailed weather information for a user's predefined city or town. Weather forecasts are read out for up to one week in advance. Forecast information, days high and days low are given for each day. Users may customize their city to suit their individual preferences and travel arrangements. RVAD allows the weekly weather forecast to be read out without user intervention.

- vNews Service - Live Rich Site Summary (RSS) News feeds can be heard at a preferred occasion based on a user's preference. The vNews service reads out each news item as requested by the users telephone key tone interaction or automatically.

\section{Conclusion}

Widespread use of mobile computing devices, telephones along with Internet services has resulted in a broad range of ways to access information and to communicate. However, true voice and data convergence has been isolated to certain environments. Converged services that are accessible anywhere and anyhow promise increased productivity, reduce costs, and new opportunities and revenues for enterprises. A versatile approach is required to produce a solution to this problem. This paper presents an Interactive Voice Response (IVR) driven solution that uses a reconfigurable plug and play software architecture and platform to dynamically compose, integrate and tailor voice access to data services. This combination reduces development and integration complexity, effort and cost while enhancing service effectiveness, flexibility and convenience for professionals on the move.

\section{ACKNOWLEDGEMENTS}

This research was partly funded by the Australian Research Council. 


\section{REFERENCES}

[1] C. R. Strathmeyer, "An Introduction to Computer Telephony", IEEE Commun. Mag., 35(5), May 1996, pp. 106-11.

[2] B. Benner, "Computer Telephony Integration (CTI) Industry", On-line at: http://faculty.ed.umuc.edu/ meinkej/inss690/benner/CTIpa per.htm (2006)

[3] F. Bronsard, D. Bryan, W. Kozaczynski, E. Liongosari, J. Ning, A. Olafsson, J. Wetterstrand, "Toward Software Plug-and-Play", In Proceedings of the Symposium on Software Reusability, Boston, Massachusetts, May 1997.

[4] A. Talevski, E. Chang, T. Dillon, "A Dynamic Meta Model Driven Framework for Integration and Extension of Underlying Application Components", In Proceedings of the International Workshop on Object-oriented Real-time Dependable Systems, Capri Island, Italy, October 2003.

[5] G. Booch, Object-Oriented Analysis and Design with Applications (Second Ed.), Benjamin / Cummings, Redwood City, Calif, 1994.

[6] A. Talevski, E. Chang, "A dynamically re-configurable component-based architecture", International Journal of Engineering and Intelligent Systems, 10(1), March 2002.

[7] R. Schutte, "SOA is changing software", On-line at: http://www4.gartner.com/resources/

111900/111987/111987.pdf (2003).

[8] M. Aoyama, "New Age of Software Development: How Component-Based Software Engineering Changes the way of Software Development", In Proceedings of the International Workshop on Component-Based Engineering, Kyoto, Japan, April 1998

[9] L. Williams, C. Smith, "Performance Evaluation of Software Architectures", In Proceedings of the Workshop on Software and Performance, Santa Fe, New Mexico, October 1998.

[10] D. Rine, N. Nada, "Software Reuse Reference Model: Development and Validation", In Proceedings of the International Conference on Software Reuse, Victoria, Canada, June 1998.

[11] N, Medvidovic, R. Taylor, "Separating Fact from Fiction in Software Architecture", Proceedings of the International Software Architecture Workshop, Orlando, Florida, USA, November 1998

[12] D. Rine, N. Nada, K. Jaber, "Using Adapters to Reduce Interaction Complexity in Reusable Component-Based Software Development", Proceedings of the Symposium on Software Reusability, Los Angeles, CA, USA, May 1999.

[13] S. Phil, F. Cary, "You Don't Know Jack About VoIP", Queue, 2004, 2(6), p. 30-38.

[14] W. Stallings, Data and Computer Communications (Seventh Ed.), Pearson Educational International, 2004

[15] Deloitte, "Getting off the Ground: Why the move to VoIP is a decision for all CXOs", On-line at: http://www.deloitte.com/dtt/research/0,1015,sid\%3D2245\& cid\%3D64027,00.html (2004)

[16] M. Grant, "Voice Quality Monitoring for VolP Networks", 2005: Melbourne.

[17] "Asterisk Home", On-line at: http://www.asterisk.org/ (2006)

[18] "AT\&T TTS", On-line at:
http://www.research.att.com/viewProject.cfm?prjID=315 (2006)

[19] "Speex Home", On-line at: http://www.speex.org/ (2006)

[20] "Hibernate Home", On-line at: http://www.hibernate.org/ (2006)

[21] "MySQL Home", On-line at: http://www.mysql.com/ (2006)

[22] "Java Sun Home", On-line at: http://java.sun.com/ (2006) 